\title{
Intraoperative electrophysiological monitoring for C1-2 spinal cord stimulation
}

\author{
Laura M. Muncie, MS, PA-C, ${ }^{1}$ Nathaniel R. Ellens, BA, ${ }^{2}$ Emeline Tolod-Kemp, MS, ${ }^{1}$ \\ Claudio A. Feler, MD, ${ }^{3,4}$ and John S. Winestone, MD ${ }^{1,2,5}$ \\ ${ }^{1}$ Great Lakes Neurosurgical Associates, Grand Rapids; ${ }^{2 M i c h i g a n ~ S t a t e ~ U n i v e r s i t y ~ C o l l e g e ~ o f ~ H u m a n ~ M e d i c i n e, ~ G r a n d ~ R a p i d s, ~}$ \\ Michigan; ${ }^{3}$ Department of Neurosurgery, Cheyenne Regional Medical Center, Cheyenne, Wyoming; ${ }^{4}$ Department \\ of Neurosurgery, University of Tennessee Medical Center, Memphis, Tennessee; and ${ }^{5}$ Shaare Zedek Medical Center, \\ Jerusalem, Israel
}

OBJECTIVE This study is a retrospective case series involving C1-2 spinal cord stimulation in patients with complex regional pain syndrome (CRPS) under general endotracheal anesthesia. Currently, C1-2 paddle lead placement is an accepted practice, which provides effective cervical stimulation to ameliorate upper-extremity and sometimes lowerextremity symptoms experienced by patients with CRPS. However, this technique must be performed under general endotracheal anesthesia rather than in an awake or semiconscious state due to intraoperative safety concerns and patient comfort. The authors aim to provide additional data to support the following novel technique: the use of somatosensory evoked potential (SSEP) diminution data to assist with proper midline placement of C1-2 leads under general anesthesia.

METHODS SSEP median nerve (MN) and posterior tibial nerve (PTN) data were collected from 6 patients undergoing placement of C1-2 leads under general anesthesia. Fluoroscopy was used as an initial guide for proper anatomical midline placement. This was followed by the activation of the spinal cord stimulator and simultaneous collection of primarily MN SSEPs as well as PTN SSEPs for physiological midline placement. Unilateral and bilateral reductions in SSEPS assisted with the correct lateralization of the lead to ensure effective postoperative coverage according to the patient's individual preoperative symptoms.

RESULTS Six patients were monitored using SSEPS and repeatable, reliable MN and PTN baseline responses were obtained from all. A reduction in amplitude ranging from $5 \%$ to $87 \%$ was observed, confirming inhibition of dorsal column conduction, and an average pain relief of $63 \%$ at short-term and $64 \%$ at long-term follow-up was recorded with 6 of 6 and 5 of 6 patients responding, respectively.

CONCLUSIONS Intraoperative SSEP collision study testing appears to be a safe technique to monitor placement of C1-2 paddle leads intraoperatively under general anesthesia.

https://thejns.org/doi/abs/10.3171/2016.7.SPINE16103

KEY WORDS complex regional pain syndrome; paddle lead; C-1; C-2; stimulation; somatosensory evoked potential; cervical

$\mathrm{C}$ OMPLEX regional pain syndrome (CRPS) is a neuropathic pain condition characterized by disturbances of sensory, motor, and autonomic function that may be associated with trophic changes. ${ }^{8}$ Classic symptoms include intractable burning pain often exacerbated by emotional distress, light touch, movement of the affected extremity, or changes in temperature ${ }^{8}$ CRPS I is defined as pain only, while CRPS II is defined as pain with neurological deficit. ${ }^{24}$
Spinal cord stimulation (SCS) is a surgical technique used to treat chronic pain disorders such as CRPS. ${ }^{719}$ This neuromodulatory technology involves the administration of therapeutic doses of electrical stimulation to the spinal cord for the management of neuropathic pain. ${ }^{5}$ Stimulation of the dorsal columns has been shown to reduce the perception of pain, with common accompaniment of paresthesia coverage in associated dermatomes. ${ }^{1,9,10}$ Common indications in addition to CRPS include failed back sur-

ABBREVIATIONS CRPS = complex regional pain syndrome; EMG = electromyography; GABA = $\gamma$-aminobutyric acid; LE = lower extremity; MN = median nerve; PTN = posterior tibial nerve; SCS = spinal cord stimulation; SSEP = somatosensory evoked potential; TO4 = train of four; UE = upper extremity.

SUBMITTED January 19, 2016. ACCEPTED July 11, 2016.

INCLUDE WHEN CITING Published online September 30, 2016; DOI: 10.3171/2016.7.SPINE16103. 
gery syndrome, diabetic neuropathy, ischemic limb pain, angina, and some forms of visceral pain., ${ }^{1,5,18}$

SCS aimed at decreasing pain in the upper extremities (UEs) typically requires placement of stimulation electrodes within the cervical spine. ${ }^{9}$ Coverage for lower-extremity (LE) symptoms is most commonly achieved with the placement of electrodes in the thoracic spine. Hayek et al. noted clinical experience and a few case reports suggestive of UE and LE coverage with SCS via percutaneous leads placed in the epidural space of the lower cervical spine. 9

In recent years, technological advancements have led to the use of intraoperative neurophysiological monitoring during cases involving the placement of spinal cord stimulators under general anesthesia. Two common neuromonitoring options exist. One method includes the use of SSEP collision studies. ${ }^{3}$ A second approach involves spontaneous electromyography (EMG) testing., ${ }^{1,6,16}$ Both approaches aim to assist the surgical team in locating the physiological midline and rostrocaudal position to ensure the proper placement of the spinal cord stimulator with the patient asleep. The ultimate goal is to increase the rate of successful postoperative paresthesia coverage for patients, therefore leading to improved clinical outcomes.

Although the current literature discusses the use of intraoperative monitoring to guide the positioning of various spinal cord stimulator leads, this paper describes the use of SSEP collision data to guide the effective placement of C1-2 paddle leads under general anesthesia. Our novel technique included a single-stage procedure in which a lead (Penta, St. Jude Medical) with a 20-contact electrode array was placed intraoperatively, with the assistance of SSEP monitoring, to confirm the physiological midline and stimulation efficacy. The SSEP collision data were used to suggest clinically relevant postoperative paresthesia coverage.

\section{Methods \\ Study Population}

Six patients with the primary diagnosis of CRPS underwent the placement of retrograde C1-2 Penta leads by the lead surgeon (J.S.W.). All of the patients were previously managed by a pain clinic physician, but it was determined that their pain was refractory to conservative and medical therapies. Preoperative imaging/testing, including MRI or CT myelography, was ordered to rule out spinal canal or nerve root compression prior to stimulator placement. Of the 6 patients, 5 were male and 1 was female. The patients' ages ranged from 38 to 73 years old. Two patients underwent successful percutaneous trials in the subaxial cervical spine. Two other patients had previously documented relief with subaxial cervical SCS. The last 2 patients had relative contraindications for trialing (e.g., previous laminectomy, subaxial stenosis, or previous subaxial implant with epidural scar).

\section{Surgical Procedure}

The procedures were performed under general endotracheal anesthesia, with the patients positioned prone in a Mayfield frame. Perioperative antibiotics were adminis- tered. Reliable SSEP baselines were obtained with median nerve (MN) SSEPs stimulated bilaterally at the wrists and posterior tibial nerve (PTN) SSEPs stimulated bilaterally at the ankles. The following anesthetic regimen was requested: $<1.0$ minimum alveolar concentration inhalation agents with as-needed supplemental narcotic boluses and/ or intravenous infusions (preferred over bolus), no $\mathrm{N}_{2} \mathrm{O}$, and 0-2/4 train-of-four (TO4) monitoring to reduce muscle artifact. The TO4 ranged from $0 / 4$ to $4 / 4$ during our studies. The surgical technique included exposure of the suboccipital area to the posterior cervical spine, subperiosteal dissection at C-1 and C-2, exposure of the epidural space, and if needed, small posterior C-1 and C-2 laminotomies to expose the epidural space. After achieving adequate exposure, the paddle lead was then passed in a rostrocaudal fashion, under direct visualization, onto the dorsal epidural space at C1-2.

A fluoroscopic image was used for the initial confirmation of midline placement of the paddle lead (Fig. 1). Once the paddle lead was in place, it was interrogated and then activated to initiate SCS with the following initial parameters: pulse width $300 \mathrm{msec}$, frequency $60 \mathrm{~Hz}$, and stimulation intensity of $2 \mathrm{~mA}$. The intraoperative paddle lead stimulation parameters ranged from a pulse width of 250-300 msec; frequency of $60,90,120$, or $160 \mathrm{~Hz}$; and stimulation intensity of $0.5-5 \mathrm{~mA}$. Differing locations of anode and cathode stimulation on the 20-lead contact were also used for lateralization and efficacy.

We simultaneously asked the intraoperative monitoring technician to begin acquiring traces of MN SSEPs and, in some instances, PTN SSEPs to evaluate any decreases in amplitude from baseline. This allowed us to confirm laterality/midline placement to ensure proper position of the lead. Once anatomical, fluoroscopic, and electrophysiological parameters were evaluated to confirm proper position of the electrodes, the remainder of the case proceeded in a standard fashion. The electrode was anchored, tunneled, and connected to the generator, and standard closure with antibiotic irrigation was performed.

\section{Results}

Upper- and lower-extremity SSEP monitoring was routinely performed during our posterior cervical surgeries. The collection of additional collision data with the spinal cord stimulator was completed within 5 minutes and not believed to add significant time to the case.

All of our patients suffered from pain syndromes involving their UEs; therefore, MN SSEP collision studies were used in all 6 cases. Two of our patients complained of LE pain in addition to UE pain preoperatively, and 1 of these patients had back pain as well. PTN SSEP collision data were collected on 4 of the 6 cases, including the 2 with LE symptoms. Reproducible SSEP baselines were obtained prior to collision testing. No new postoperative deficits occurred in any of these patients and no additional significant changes were observed in the SSEP traces other than the transient changes observed while conducting the collision studies. Reliable SSEP blocking was observed in every case (Fig. 2).

The average hospital stay was 2-3 days, including the day of surgery, and the stimulators were turned on to trial 


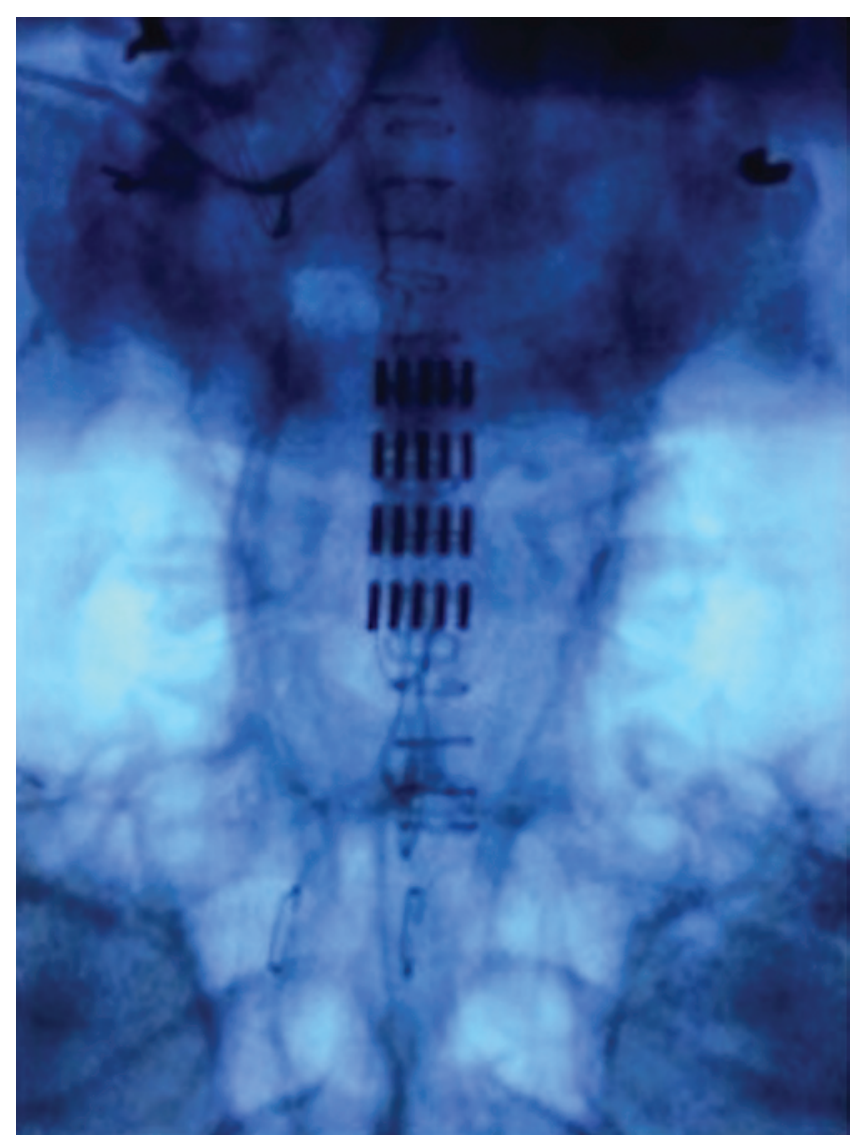

FIG. 1. Intraoperative fluoroscopic image of an implanted C1-2 St. Jude Penta lead. Figure is available in color online only.

programs the first postoperative day. Patients returned to the surgeon (J.S.W.) 2 weeks postoperatively for followup. With the exception of surgical or implant complications, future care was managed by the referring pain clinic physician. Patients were asked to rate their pain relief as a percentage of pain reduction at their 2-week postoperative visit, and again by telephone at approximately 2 years postoperatively (range 23-31 months, 5 of 6 responders, mean 25.2 months).

Relief at 2 weeks ranged from $50 \%$ to $80 \%$, with an average of $63 \%$. At 2 years, 5 of 6 patients had responded, and relief ranged from $40 \%$ to $80 \%$, with an average of $64 \%$. One patient had a superficial wound infection that resolved with antibiotics. A second patient had a distal hardware malfunction after a fall and underwent a distal revision that restored stimulation at 23 months (Table 1).

\section{Discussion}

SCS for the treatment of pain was introduced in 1967.5 Since its development, SCS has been shown to provide consistent, long-term pain relief and improved quality of life in patients with chronic pain. ${ }^{14,15}$ Additionally, SCS has been shown to be more effective than reoperation for the treatment of chronic pain related to disc herniation. ${ }^{19}$ Subsequently, both percutaneous and paddle leads have been placed in the epidural space of the spinal cord within the cervical and thoracic spine using various techniques (awake vs asleep) and methods to confirm proper placement: direct patient feedback, fluoroscopy, and neurophysiological monitoring (SSEP/EMG).

Although the mechanism of SCS is still not completely understood, its effects may be related to the gate theory, proposed by Melzack and Wall in $1965 .{ }^{17}$ This theory suggests that the substantia gelatinosa may modulate afferent pain transmission, and its proposal generated an interest in neurosurgical treatment for chronic pain. ${ }^{17}$ Yampolsky et al. suggested that SCS may selectively influence transmission of Type A fibers, as observed in rat studies, based on antidromic activation of dorsal column tracts. Activation of inhibitory interneurons in or near the substantia gelatinosa and marginal layer of the dorsal horn inhibits the transmission of pain. ${ }^{24}$ Pain relief may also be, in part, related to the restoration of normal $\gamma$-aminobutyric acid (GABA) levels in the dorsal horns of the spinal cord and an increase in the release of adenosine. ${ }^{24}$ Studies in nervelesioned rats also show that SCS results in an increased release of GABA in the dorsal horn, resulting in a decrease in the excitatory amino acids glutamate and aspartate. ${ }^{7}$ Issa and Kim proposed an additional theory of modulation of the autonomic nervous system as demonstrated in animal studies, involving activation of the descending inhibitory system, antidromic activation of sensory nerves (A-delta and $\mathrm{C}$ fibers), and the subsequent release of vasodilators, such as calcitonin gene-related peptide and nitric oxide. ${ }^{11}$ This theory of autonomic modulation correlates well with the resolution of autonomic changes of CRPS with SCS.

Precise electrode positioning relative to the spinal cord is critical for successful stimulation. Barolat et al. propose that applying an electrical field to the dorsal epidural space may activate large afferent myelinated fibers, therefore most likely involving the dorsal columns, dorsal roots, dorsal root entry zone, and dorsal horns. ${ }^{4}$ Their work includes the mapping of sensory responses to epidural stimulation using electrical activation of the dorsal root/dorsal column complex at various vertebral levels. Contacts placed within $3 \mathrm{~mm}$ of radiological midline elicited more responses from the dorsal columns and laterally placed electrodes commonly provided paresthesia, likely generated from the dorsal roots. They found that stimulation of the median aspect of the hand could be elicited from a large percentage of combinations with electrodes placed in the cervical spine, and also found that the region of the body that carried the highest likelihood of inducing paresthesia from SCS was within the feet. In this study, paresthesia coverage of the feet was achieved to varying degrees from leads placed in the thoracic/lumbar and midline cervical spine. ${ }^{4}$

Falowski et al. describe the somatotopic response pattern of SCS at varying levels. ${ }^{5}$ The posterior occiput and C-2 occasionally correlate with the lower jaw, C2-4 correlate with the shoulder, C5-6 with the hand, C-7 with the anterior chest wall, and T11-L1 with the posterior thigh and foot. When placed midline, T8-9 correlate with the low back (difficult to obtain) and when placed laterally, T11-12 correlate with the anterior thigh. ${ }^{5}$ Stimulation of dorsal column fibers generates paresthesia coverage to several dermatomes caudal to the level of the stimulating cathode. ${ }^{18}$ Preferential stimulation of the dorsal columns is achieved with narrow spacing between electrodes. Mol- 


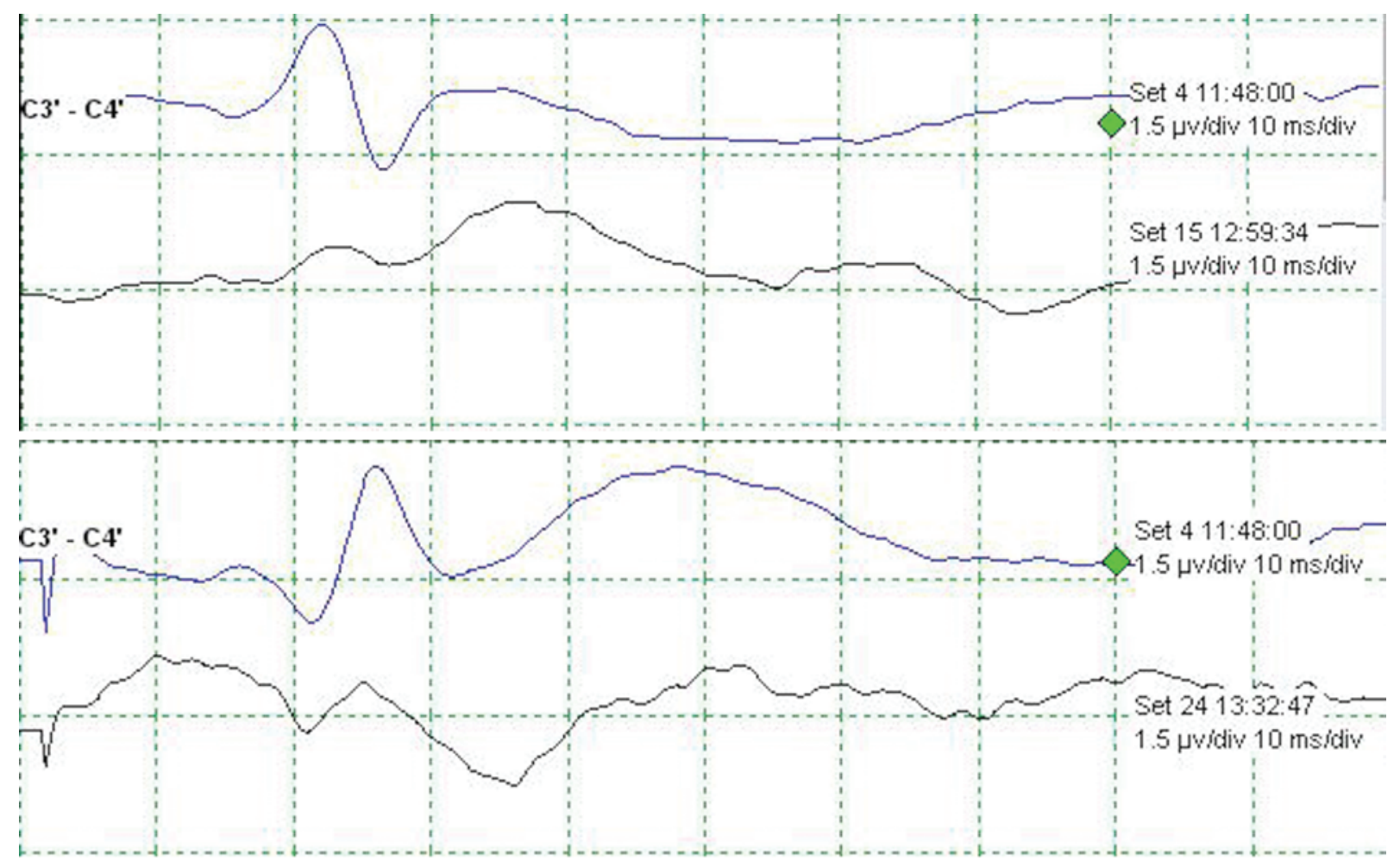

FIG. 2. Collision data. These figures provide an example of the paddle lead coverage of Case 1. The MN was stimulated, producing a cortical response that is measured at the $\mathrm{C}^{\prime}-\mathrm{C}^{\prime}$ electrode placed behind the central sulcus on the scalp. These figures indicate the latency in milliseconds (x-axis) and amplitude in microvolts (y-axis) of the cortical response with (set 24 and set 15) and without (set 4) activation of the spinal cord stimulator. Upper: Right MN decrease with simultaneous C1-2 paddle lead stimulation (set 15) C3'-C4' montage. Set 4 (at 11:48) represents the preoperative baseline. Set 15 (at 12:59) represents the activated stimulator demonstrating an amplitude reduction of $27 \%-87 \%$. Lower: Left MN decrease with simultaneous $\mathrm{C} 1-2$ paddle lead stimulation (set 15) C3'-C4' montage. Set 4 (at 11:48) represents the preoperative baseline. Set 24 (at 13:32) represents the activated stimulator demonstrating an amplitude reduction of $8 \%-42 \%$. Figure is available in color online only.

nar and Barolat suggest that bipolar (cathode with adjacent anode) or guarded cathode (flanked rostrally and caudally by an anode), and transverse tripolar (cathode flanked laterally by anodes) configurations are preferred to achieve optimal stimulation of the desired neural element (dorsal columns), while avoiding elements that would result in side effects or undesirable clinical outcomes. ${ }^{18}$ Hence, common practice today is stimulation to the lower thoracic cord for LE coverage, and to the cervical cord for UE coverage, 1-2 vertebral levels above the targeted dermatomal level.

Both percutaneous and paddle leads may be used for SCS. Percutaneous leads are wirelike, flexible implants with electrodes in series, with 6-8 contacts. They may be inserted via Tuohy needle for both trial and permanent implantations. Conversely, paddle leads require a small laminotomy to be introduced into the epidural space and therefore are usually limited to permanent implantation. Although more effective, paddle lead electrode placement does require a more invasive implantation. ${ }^{5}$ Advantages of paddle leads include increased stability in the dorsal epidural space, as well as increased stimulatory options, with multiple contacts in both rostrocaudal and mediolateral planes. One example of this type of lead is the Penta lead, used in our study. Disadvantages of percuta- neous leads include lead migration, inherent flexibility, decreased energy efficiency (due to greater current shunting), and increased patient complaints of positional variance in paresthesia coverage when compared with paddle electrodes. ${ }^{5}$

There is recent data indicating better long-term durability with paddle implants due to decreased risk of lead migration and lower reoperation rates when compared with percutaneous lead placement. ${ }^{2}$ Additionally, paddle leads are associated with significantly greater long-term pain relief than percutaneous leads. ${ }^{22}$ Paddle leads also have the advantage of larger spatial distribution of contacts in both rostrocaudal and mediolateral planes, increasing the chance of successful localization.

Several strategies may be used for optimizing localization. A trial lead, usually percutaneous, may be implanted and then connected to an external generator for several days. This can confirm response to stimulation at the designated level, and is a good predictor for long-term response with a permanent, often paddle lead, at that same site. ${ }^{5}$ A second strategy involves intraoperative evaluation and trialing of the permanent lead. The implantation of the permanent lead is performed under local anesthesia with sedation. Once the lead is in position, the patient is woken up and coverage is evaluated. 
TABLE 1. Summary of the stimulator coverage and pain relief of each patient in our series

\begin{tabular}{|c|c|c|c|c|c|c|c|c|c|c|}
\hline $\begin{array}{l}\text { Case } \\
\text { No. }\end{array}$ & $\begin{array}{l}\text { Age } \\
\text { (yrs), } \\
\text { Sex }\end{array}$ & $\begin{array}{l}\text { Trial/ } \\
\text { Previous } \\
\text { SCS }\end{array}$ & Diagnosis & $\begin{array}{c}\text { Pain } \\
\text { Distribution }\end{array}$ & $\begin{array}{c}\text { Reduction } \\
\text { Amplitude of } \\
\text { SSEPs in MN (\%) }\end{array}$ & $\begin{array}{l}\text { Coverage } \\
\text { Results } \\
\text { at FU }\end{array}$ & $\begin{array}{l}\text { Initial } \\
\text { Relief } \\
(\%)\end{array}$ & $\begin{array}{l}\text { Long- } \\
\text { Term FU } \\
\text { (mos) }\end{array}$ & $\begin{array}{c}\text { Relief } \\
(\%)\end{array}$ & $\begin{array}{l}\text { Additional } \\
\text { Treatment }\end{array}$ \\
\hline 1 & $54, \mathrm{~F}$ & SCS & CRPS I & UE/LE & Rt 27-87, It 8-42 & Neck, bilat UE & 50 & 31 & 50 & None \\
\hline 2 & $38, M$ & Trial & CRPS II & UE & Rt $60-80$, It $20-30$ & Rt UE & 60 & NA & NA & Local infection, antibiotics \\
\hline 3 & $59, M$ & SCS & CRPS ॥ & UE/LE & Rt $14-55$, It $66-70$ & Neck, bilat UE, back & 50 & 25 & 65 & None \\
\hline 4 & $63, M$ & Trial & CRPS II & UE & Rt 11-29, It 27-51 & Rt UE, rt LE & 60 & 24 & $40-50$ & None \\
\hline 5 & $73, \mathrm{M}$ & No & CRPS II & UE & Rt $12-31$, It 5-51 & Lt UE & 80 & 23 & 80 & $\begin{array}{l}\text { Distal revision at } 22 \text { mos } \\
\text { after fall }\end{array}$ \\
\hline 6 & $62, \mathrm{M}$ & No & CRPS II & UE & Poor signal & Lt UE & 80 & 23 & 80 & None \\
\hline
\end{tabular}

$\mathrm{FU}=$ follow-up; $\mathrm{NA}=$ not available.

None of the patients had LE coverage with SCS.

Traditionally, electrodes in the thoracolumbar spine were placed during the administration of local anesthesia and conscious sedation, allowing for patient communication in the intraoperative setting. This allowed the patient to confirm that painful regions were adequately covered. ${ }^{3}$ Furthermore, intraoperative imaging with fluoroscopy is often used to confirm proper placement, although image quality may vary and the ability to determine true midline may decrease when traditional markers such as spinous processes are missing. The relationship between the spinal cord midline and the vertebral column midline can also vary.

Intraoperative neurophysiological monitoring has been described as an additional technique to confirm proper coverage. Currently there are 2 techniques that may be used during open implantation of SCS: SSEP and EMG. Via feedback data, SSEPs assist with optimizing placement and ensuring the inhibition of the dorsal columns of the spinal cord. SSEP signals are "blocked" when stimulation is activated at the dorsal columns. Drawbacks include increased time necessary to average signals, increased anesthesia burden to maintain relatively low inhalational agents, and susceptibility to electrical artifact (especially PTN). SSEPs can successfully predict the lateralization of an epidural electrode and the corresponding stimulationinduced paresthesias in patients undergoing SCS at the cervical level. ${ }^{3}$

When compared with SSEP monitoring, EMG provides a more immediate feedback response because it does not require signal averaging. EMG can also define laterality during stimulator placement and may be used with higher levels of inhalational agents. However, despite these important advantages, EMG requires 4/4 TO4 to be considered reliable, increasing the likelihood of patient movement during the case. The patient also remains susceptible to electrical artifact and, most importantly, EMG does not confirm dorsal column inhibition. ${ }^{16}$ Our study relied on SSEPs to minimize safety concerns of cases conducted with the patient positioned prone, in a fixed Mayfield head frame.

Recently, thoracic electrode implantation has been performed under general anesthesia. This may be useful for patients who cannot tolerate a prone procedure in a semiconscious state, including procedures requiring a more extensive laminectomy, scarring in the epidural space from prior procedures, medical comorbidities, allergies to local anesthesia, or communication barriers. ${ }^{1,6}$ Falowski et al. suggests that the incidence of device failure for patients whose leads were placed using electrophysiological monitoring (intraoperative EMG) was lower when compared with patients receiving lead placement via sedation and intraoperative trial. ${ }^{6}$ Balzer et al. incorporated the use of SSEP monitoring to assist with the localization of SCS leads in both the cervical and cervicomedullary spine. In this study, SSEP responses with an amplitude reduction of greater than or equal to $75 \%$ were indicative of lateralized placement or a bilateral reduction at or near midline placement. ${ }^{3}$

Neurophysiological monitoring may be particularly useful in the cervical spine, as conditions requiring stimulation in the cervical spine pose challenges for traditional localization techniques. For example, staged percutaneous trials may not be possible in patients with prior cervical surgery, due to epidural scarring. Additionally, an intraoperative trial of a cervical paddle lead is often not feasible, given that general anesthesia is preferred, along with rigid head fixation in the prone position.

The efficacy and safety of neurophysiological monitoring has been reported with thoracic and cervical spinal cord stimulator implantation. However, this is the first dedicated report of using monitoring during surgery at C1-2 for single-stage paddle-lead spinal cord stimulator implantation.

The limitations of percutaneous electrodes and the narrow diameter of the lower cervical spine led to the implantation of sublaminar $\mathrm{Cl}-2$ paddle leads that have been proven to be a safe and effective way of treating neuropathic pain in the upper extremities. ${ }^{23}$ Whitworth and Feler first described the advantage of using the increased space at the $\mathrm{C} 1-2$ level, which is rarely stenotic or scarred from previous surgery. Anteroposterior canal diameter of the cervical spinal cord is $23 \mathrm{~mm}$ at C-1, $20 \mathrm{~mm}$ at C-2, 17-18 $\mathrm{mm}$ at $\mathrm{C} 3-6$, and $15 \mathrm{~mm}$ at $\mathrm{C}-7$, making the rostral cervical spine a logical option for implant placement. Retrograde placement of a dorsal column lead at $\mathrm{C} 1-2$ provided good coverage to patients with UE complaints. Given the need for prone position, pin fixation of the head, airway concerns, and the rostral cervical/suboccipital open exposure, general anesthesia was used. ${ }^{23}$

This study was a retrospective case series, limited by a small number of patients. Long-term follow-up was 
also limited to only $23-31$ months postoperatively, with an average of 25 months postoperatively. While patients were queried regarding postoperative versus preoperative percentage pain reduction, health-related quality of life instruments were not used. Also, it could be argued that using anatomical placement with radiological confirmation without SSEP blocking confirmation is comparably effective in providing postoperative paresthesia. However, given the frequent use of $\mathrm{C} 1-2$ placement as a revision technique, and the accepted standard of intraoperative interrogation, intraoperative neuromonitoring, or staged trialing at other locations in the spinal column, our technique is a reasonable consideration.

Intraoperative SSEP collision studies added minimal time and risk. The signal averaging time was insignificant, yet it assisted us with the confirmation of a decrease in the localized response to confirm laterality, thereby identifying the physiological midline of the spinal cord. This allows for the adjustment of intraoperative paddle lead placement, and the adjustment of the paddle lead stimulation montage to predict coverage of the target extremity or extremities. Responses returned to baseline with the stimulator turned off. In this series no significant changes in electrode placement were made based on SSEP collision studies alone. Outcome was not influenced, and costbenefit analysis of this series does not justify the expense of intraoperative neurophysiological monitoring. It is conceivable to have cases in which anatomical, radiographic, and neurophysiological data may contradict each other in their suggestion of optimal electrode placement. While it is logical to hypothesize that the neurophysiological data are most reliable, further studies are needed to evaluate cost-benefit ratio, as well as how to use neurophysiological monitoring in deciding upon electrode placement in cases with conflicting data of optimal placement.

Effective cervical stimulation covering UEs and LEs has been reported in other studies. ${ }^{9,12,13,20,21,23}$ Theoretically, MN blocking should correlate with UE relief and PTN blocking should correlate with LE relief. However, from the 2 patients in our study who had LE symptoms, neither had effective long-term LE coverage.

More investigation is needed regarding the mechanism of cervical stimulation in treating UE and LE symptoms in patients with CRPS. Using 1 stimulator and battery is clearly preferable to separate systems for UEs and LEs. Four-limb CRPS may be more effectively treated with a single device. Intraoperative neurophysiological monitoring may be useful when attempting to cover 4 extremities. $\mathrm{Cz}^{\prime}$-Fpz scalp electrode placement may be a better montage to watch for LE involvement via reduction of PTN SSEP. In our study, $0.5 \mathrm{~mA}$ was applied to the paddle lead on one occasion (LE movement at higher stimulation) in a patient with LE complaints. This may also, in turn, further develop understanding of CRPS pathophysiology. It is currently unknown which structures have the most impact on pain relief.

In summary, further clinical studies may assess developing options for percutaneous intervention; cost-benefit analysis specifically for neurophysiological monitoring; comparing reliability of anatomical, radiographic, and neurophysiological parameters; evaluating SSEPs versus
EMG; and defining the role of neurophysiological monitoring in attempting 4-limb coverage.

\section{Conclusions}

Intraoperative use of SSEPs in the placement of C1-2 SCS leads appears safe and useful in the treatment of chronic pain. Further investigation will be necessary for validation as well as developing understanding of the mechanism of action of SCS, potentiating more effective treatment.

\section{References}

1. Air EL, Toczyl GR, Mandybur GT: Electrophysiologic monitoring for placement of laminectomy leads for spinal cord stimulation under general anesthesia. Neuromodulation 15:573-580, 2012

2. Babu R, Hazzard MA, Huang KT, Ugiliweneza B, Patil CG, Boakye M, et al: Outcomes of percutaneous and paddle lead implantation for spinal cord stimulation: a comparative analysis of complications, reoperation rates, and health-care costs. Neuromodulation 16:418-427, 2013

3. Balzer JR, Tomycz ND, Crammond DJ, Habeych M, Thirumala PD, Urgo L, et al: Localization of cervical and cervicomedullary stimulation leads for pain treatment using median nerve somatosensory evoked potential collision testing. J Neurosurg 114:200-205, 2011

4. Barolat G, Massaro F, He J, Zeme S, Ketcik B: Mapping of sensory responses to epidural stimulation of the intraspinal neural structures in man. J Neurosurg 78:233-239, 1993

5. Falowski S, Celii A, Sharan A: Spinal cord stimulation: an update. Neurotherapeutics 5:86-99, 2008

6. Falowski SM, Celii A, Sestokas AK, Schwartz DM, Matsumoto C, Sharan A: Awake vs. asleep placement of spinal cord stimulators: a cohort analysis of complications associated with placement. Neuromodulation 14:130-135, 2011

7. Forouzanfar T, Kemler MA, Weber WE, Kessels AG, van Kleef M: Spinal cord stimulation in complex regional pain syndrome: cervical and lumbar devices are comparably effective. Br J Anaesth 92:348-353, 2004

8. Grabow TS, Tella PK, Raja SN: Spinal cord stimulation for complex regional pain syndrome: an evidence-based medicine review of the literature. Clin J Pain 19:371-383, 2003

9. Hayek SM, Veizi IE, Stanton-Hicks M: Four-limb neurostimulation with neuroelectrodes placed in the lower cervical epidural space. Anesthesiology 110:681-684, 2009

10. Holsheimer J, Khan YN, Raza SS, Khan EA: Effects of electrode positioning on perception threshold and paresthesia coverage in spinal cord stimulation. Neuromodulation 10:34-41, 2007

11. Issa MA, Kim CH: Cervical spinal cord stimulation with 5-column paddle lead in Raynaud's disease. Pain Physician 15:303-309, 2012

12. Kapural L, Cywinski J, Zovkic P, Stanton-Hicks M: Effective cervical spinal cord stimulation for bilateral lower extremities pain: technical report. Pain Pract 8:117-119, 2008

13. Kim JK, Hong SH, Lee JK: High-level cervical spinal cord stimulation used to treat intractable pain arising from transverse myelitis caused by schistosomiasis. J Korean Neurosurg Soc 47:151-154, 2010

14. Kumar K, Hunter G, Demeria D: Spinal cord stimulation in treatment of chronic benign pain: challenges in treatment planning and present status, a 22-year experience. Neurosurgery 58:481-496, 2006

15. Kumar K, Taylor RS, Jacques L, Eldabe S, Meglio M, Molet J, et al: Spinal cord stimulation versus conventional medical management for neuropathic pain: a multicentre randomised controlled trial in patients with failed back surgery syndrome. Pain 132:179-188, 2007 
16. Mammis A, Mogilner AY: The use of intraoperative electrophysiology for the placement of spinal cord stimulator paddle leads under general anesthesia. Neurosurgery 70 ( 2 Suppl Operative):230-236, 2012

17. Melzack R, Wall PD: Pain mechanisms: a new theory. Science 150:971-979, 1965

18. Molnar G, Barolat G: Principles of cord activation during spinal cord stimulation. Neuromodulation 17 (Suppl 1):12-21, 2014

19. North RB, Kidd DH, Farrokhi F, Piantadosi SA: Spinal cord stimulation versus repeated lumbosacral spine surgery for chronic pain: a randomized, controlled trial. Neurosurgery 56:98-107, 2005

20. Simpson BA, Bassett G, Davies K, Herbert C, Pierri M: Cervical spinal cord stimulation for pain: a report on 41 patients. Neuromodulation 6:20-26, 2003

21. Vallejo R, Kramer J, Benyamin R: Neuromodulation of the cervical spinal cord in the treatment of chronic intractable neck and upper extremity pain: a case series and review of the literature. Pain Physician 10:305-311, 2007

22. Villavicencio AT, Leveque JC, Rubin L, Bulsara K, Gorecki JP: Laminectomy versus percutaneous electrode placement for spinal cord stimulation. Neurosurgery 46:399-406, 2000

23. Whitworth LA, Feler CA: C1-C2 sublaminar insertion of paddle leads for the management of chronic painful conditions of the upper extremity. Neuromodulation 6:153-157, 2003

24. Yampolsky C, Hem S, Bendersky D: Dorsal column stimulator applications. Surg Neurol Int 3 (Suppl 4):S275-S289, 2012

\section{Disclosures}

Dr. Feler is a patent holder for St. Jude Medical.

\section{Author Contributions}

Conception and design: Muncie, Feler, Winestone. Acquisition of data: Ellens, Muncie, Winestone. Analysis and interpretation of data: Ellens, Muncie, Tolod-Kemp, Winestone. Drafting the article: Ellens, Muncie, Tolod-Kemp, Winestone. Critically revising the article: all authors. Reviewed submitted version of manuscript: Ellens, Muncie, Feler, Winestone. Approved the final version of the manuscript on behalf of all authors: Ellens. Administrative/technical/material support: Ellens, Tolod-Kemp. Study supervision: Feler, Winestone.

\section{Supplemental Information}

\section{Previous Presentations}

Portions of this work were presented in poster form at Grand Rapids Medical Education Partners Research Day in Grand Rapids, Michigan, on April 22, 2015, and at the American Academy of Pain Management 26th Annual Clinical Meeting in Washington, DC, on September 18, 2015.

\section{Correspondence}

Nathaniel R. Ellens, Michigan State University College of Human Medicine, 257 141st Ave., Wayland, MI 49348. email: ellensna@ msu.edu. 